\title{
Maternal undernutrition alters the skeletal muscle development and methylation of myogenic factors in goat offspring
}

\author{
Xiaoling Zhou ${ }^{1,2,3, a}$, Qiongxian Yan ${ }^{2,4, a}$, Liling Liu', Genyuan Chen ${ }^{1}$, \\ Shaoxun Tang ${ }^{2,3}$, Zhixiong $\mathrm{He}^{2,4}$, and Zhiliang $\operatorname{Tan}^{2,5, *}$
}

\footnotetext{
* Corresponding Author: Zhiliang Tan Tel: +8673184619702, Fax: +8673184612685, E-mail: zltan@isa.ac.cn

${ }^{1}$ College of Animal Science, Tarim University, Alaer 843300, China

${ }^{2}$ Institute of Subtropical Agriculture, The

Chinese Academy of Sciences, Changsha

410125, China

${ }^{3}$ College of Resources and Environment,

University of Chinese Academy of Science,

Beijing 100049, China

${ }^{4}$ Hunan Co-Innovation Center for Utilization of Botanical Functional Ingredients, Changsha 410128, China

${ }^{5}$ Hunan Co-Innovation Center of Animal Production Safety, CICAPS, Changsha 410128, China

a These authors contributed equally to this work

ORCID

Xiaoling Zhou

https://orcid.org/0000-0001-6039-5540

Qiongxian Yan

https://orcid.org/0000-0003-0035-6819

Liling Liu

https://orcid.org/0000-0001-7741-8200

Genyuan Chen

https://orcid.org/0000-0002-9231-3842

Shaoxun Tang

https://orcid.org/0000-0001-9343-1341

Zhixiong $\mathrm{He}$

https://orcid.org/0000-0002-4802-3506

Zhiliang Tan

https://orcid.org/0000-0002-7483-1478
}

Submitted Jun 22, 2021; Revised Aug 16, 2021; Accepted Nov 8, 2021
Objective: The effects of maternal undernutrition during midgestation on muscle fiber histology, myosin heavy chain (MyHC) expression, methylation modification of myogenic factors, and the mammalian target of rapamycin (mTOR) signaling pathway in the skeletal muscles of prenatal and postnatal goats were examined.

Methods: Twenty-four pregnant goats were assigned to a control (100\% of the nutrients requirement, $n=12$ ) or a restricted group ( $60 \%$ of the nutrients requirement, $n=12$ ) between 45 and 100 days of gestation. Descendants were harvested at day 100 of gestation and at day 90 after birth to collect the femoris muscle tissue.

Results: Maternal undernutrition increased $(\mathrm{p}<0.05)$ the fiber area of the vastus muscle in the fetuses and enhanced $(\mathrm{p}<0.01)$ the proportions of MyHCI and MyHCIIA fibers in offspring, while the proportion of MyHCIIX fibers was decreased ( $\mathrm{p}<0.01$ ). DNA methylation at the +530 cytosine-guanine dinucleotide (CpG) site of the myogenic factor 5 (MYF5) promoter in restricted fetuses was increased $(\mathrm{p}<0.05)$, but the methylation of the MYF5 gene at the $+274,280 \mathrm{CpG}$ site and of the myogenic differentiation (MYOD) gene at the $+252 \mathrm{CpG}$ site in restricted kids was reduced $(\mathrm{p}<0.05)$. mTOR protein signals were downregulated $(\mathrm{p}<0.05)$ in the restricted offspring.

Conclusion: Maternal undernutrition altered the muscle fiber type in offspring, but its relationship with methylation in the promoter regions of myogenic genes needs to be elucidated.

Keywords: DNA Methylation; Goat; Intrauterine Undernutrition; Muscle Fiber Type; Myogenic Factors

\section{INTRODUCTION}

During myogenesis, the numbers of myoblasts, primary muscle fibers, and secondary muscle fibers are determined before birth in humans [1] and domestic animals [2]. Skeletal muscle is highly susceptible to nutritional deficiencies due to the low priority of nutrient acquisition in mammals $[3,4]$. Nutritional deficiency in early gestation decreases the number of primary muscle fibers [5], but the effects are generally minor due to the low nutrients requirement of early fetuses. Secondary muscle fiber proliferates from approximately $0.3 \mathrm{G}$ (30\% of gestation stage), and this process ceases at approximately $0.7 \mathrm{G}[1,2,6]$, which constitutes nearly $95 \%$ of the final fiber population [6]. Insufficient nutrition during midgestation (0.3 to $0.7 \mathrm{G}$ ) affects the number, size, and type of secondary fibers before [6,7] and after birth $[8,9]$. It is widely accepted that skeletal muscle development is nutritionally programmed in utero [3,4], and knowledge of the underlying mechanisms of this programming helps to understand the nosogenesis of some congenital myopathies, such as fiber type disproportion in humans, or to improve meat production in livestock. 
Alterations in muscle phenotype can partly be interpreted by changes in hormone response [10], the mammalian target of rapamycin (mTOR) signaling pathway [7], myogenic regulatory factor expression [11,12] and noncoding RNA [13]. However, an unresolved question is how the phenotype is programmed during ontogenetic muscle development. Epigenetic modification is one of the key mechanisms responsible for persistent programming [3]. Myogenic factors, including myogenic differentiation 1 (MYOD), myogenin (MYOG), myogenic factor 5 (MYF5), and myogenic factor 6 (MYF6), govern skeletal muscle determination and differentiation [14], and among these, MYF5 and MYOD are the master regulators. Epigenetic modification, including DNA methylation of enhancer chromatin [15], histone acetylation, and long noncoding RNA [13] of the MYF5 and MYOD genes, is associated with the expression of muscle-specific genes during myogenesis and differentiation. Methylation of the promoter region is a regulatoty target in intrauterine skeletal programming, for example methylation of the peroxisome proliferator-activated receptor gamma coactivator-1 alpha (PGC1A) promoter [16]. However, the relationship between methylation of the promoter regions of the MYF5 and MYOD genes and uscle developmental programming under nutrient stress remains uncharacterized. DNA methylation generally reduces the expression of muscle-related genes $[16,17]$. We thus hypothesized that maternal undernutrition during midgestation would decrease the expression of MYF5 and MYOD genes by increasing the methylation of their promoter regions.

Small ruminants are optimum animal models for studying intrauterine programming, and 40\% energy restriction in pregnant goats led to hepatic metabolic programming in offspring in our previous study [18]. In the present study, we investigated the effects of $40 \%$ maternal undernutrition during midgestation on muscle fiber histology, myosin heavy chain expression, methylation modification of myogenic factors, and the mTOR signaling pathway in the skeletal muscles of prenatal and postnatal goats.

\section{MATERIALS AND METHODS}

\section{Animal care}

The present experiment was reviewed and approved by the Animal Care Committee according to the Animal Care and Use Guidelines of the Institute of Subtropical Agriculture, the Chinese Academy of Sciences, Changsha, China (No. KYNEAAM-2015-0009).

\section{Experimental design and animal management}

Twenty-four goats ( $45 \pm 3$ days of gestation, Liuyang black goats, a local breed) were selected and tested by portable ultrasonography (Aloka SSD-500 with a 5-MHz linear probe; Aloka,
Shanghai, China). Dams were randomly assigned to the control (100\% of the nutrients requirements suggested in the Chinese Meat Goat Requirements [2004], $\mathrm{n}=12$ ) or restricted group ( $60 \%$ of the nutrients requirements, $n=12$ ) according to body weight (BW) and litter size. All dams were placed within individual pens and fed a diet twice $(0800 \mathrm{~h}$ and 1600 h) per day with a 50:50 ratio of concentrate to roughage, with free access to drinking water. The ingredients and composition of the experimental diet on a dry matter basis are shown in additional Supplementary Table S1. The feeding of the restricted group was conducted by providing $60 \%$ of the feed allowance of the control group from 45 to 100 days of gestation, and the actual restriction level $(1.04 \mathrm{~kg} / \mathrm{d}$ for the control group vs $0.62 \mathrm{~kg} / \mathrm{d}$ for the restricted group) was $60.2 \%$ after measurement of the daily feed allowance and refusal. At day 100 of gestation, six pregnant dams per group were selected for harvesting, and 10 fetuses (three singlets, two sets of twins, and one set of triplets) in each group were obtained. The ratio of females to males was 7:3 in the control group and 6:4 in the restricted group.

After day 100 of gestation, feed restriction was removed, and the remaining dams were fed to supply $100 \%$ of the nutrients requirements and were managed as before during the following gestation and lactation stages. The length of the gestation period was approximately 150 days. After parturition, neonatal kids were nursed by their dams until preweaning between days 50 and 60 . Preweaning was conducted by separating offspring from their dams in the daytime from 0800 to $1600 \mathrm{~h}$, and a mixed diet of starter and fresh Miscanthus spp. was provided at a ratio of 20:80 during this period. Complete weaning was performed at 60 days of age. Then, all kids were given ad libitum access to the above diet and had free access to drinking water. The ingredients and composition of the kid diet are shown in additional Supplementary Table S1. At 90 days of age, eight kids in each group were obtained for harvesting. The ratio of females to males was 3:5 in the control group and 4:4 in the restricted group.

Body weight measurement and muscle tissue sampling: At day 100 of gestation and at day 90 after birth, the feeding of all selected animals was withdrawn for $24 \mathrm{~h}$, while clear water was offered freely. Following electric shock and exsanguination, fetuses at day 100 were removed from the uterus. After the umbilical cord was cut, each fetus was immediately weighed. The empty BW of kids was reported previously [18]. Samples of semitendinosus (ST) and vastus lateralis (VL) muscle were collected from the kids, while the gross vastus muscle in the fetuses was sampled because it was difficult to dissect into individual muscles. The sliced tissue samples were immersed in $10 \%$ formalin fixing solution for histology measurement, while another aliquot of samples was quick-frozen in liquid nitrogen and then stored at $-80^{\circ} \mathrm{C}$ for further analysis. 


\section{Histology}

After fixation, the muscle samples were embedded in paraffin, sliced at a thickness of $8 \mu \mathrm{m}$ using a rotary microtome (RM2016; Leica Microsystems Inc., Wetzlar, Hesse-Darmstadt, Germany), and stained with hematoxylin (AS1055A; Aspen Biological, Inc., Wuhan, China) and eosin (AS1094; Aspen Biological, Inc., China) according to Zou et al [11]. Ten different microscopic fields of each section and five sections per sample were randomly chosen to determine the muscle fiber area using the Image-Pro Plus 6.0 software (Media Cybernetics, Bethesda, MD, USA).

\section{Quantitative real-time polymerase chain reaction}

The extraction and quantification of total RNA and the analyses of messenger RNA (mRNA) expression levels were performed using the SYBR green-based method with gene-specific primers (Table 1) according to Zhou et al [18]. A melting curve analysis was conducted to confirm specific product amplification. Actin gamma 1 (ACTG1) was used as the reference gene, and the real-time polymerase chain reaction data were calculated using the $2^{-\Delta \Delta \mathrm{Ct}}$ method. The expression of mRNA is presented as the fold change relative to the reference gene.

\section{Immunoblotting analysis}

The extraction and quantification of total protein and the immunoblotting analysis were carried out according to the method described previously [19]. The primary antibodies against MyHCI (MYH7, No. MFCD00162703; Merck KGaA, Darmstadt, Hesse-Darmstadt, Germany), MyHC-IIa (MYH2, No. ab124937; Abcam PLC, Cambridge, MA, USA), MyHC-IIx (No. BM0096; Boster Biological Technology Co., Ltd, Pleasanton, CA, USA), and $\beta$-tubulin (No. 2146S; Cell Signaling Technology, Inc., Danvers, MA, USA) were diluted 1:5,000, $1: 10,000,1: 100$, and 1:1,000, respectively. Primary antibodies against AKT serine/threonine kinase 1 (AKT, No. AV06008; Merck KGaA, Germany), p-AKT (No. 9275; Cell Signaling Technology, Inc., USA), phosphoinositide-3-kinase regulatory subunit 1 (PI3K, No. C312573; LifeSpan BioSciences, Inc., Seattle, WA, USA), p-PI3K (No. C358831; LifeSpan BioSciences, Inc., USA), mTOR (No. PLA0114; Merck KGaA, Germany), p-mTOR (No. 2971; Cell Signaling Technology, Inc., USA), and $\beta$-actin (No. 4976; Cell Signaling Technology, Inc., USA) were diluted as $1 / 1,000$. The density of bands was quantified and then normalized to the reference protein of $\beta$-tubulin or $\beta$-actin. The normalized values were used for comparison of the relative expression levels of the target proteins between the control group and the restricted group.

\section{DNA methylation detection}

Approximately $20 \mathrm{mg}$ of frozen sample was ground in liquid nitrogen, and DNA was extracted using a DNeasy Blood \& Tissue Kit (Qiagen, Hilden, Nordrhein-Westfalen, Germany) according to the manufacturer's instructions. Quantitative methylation analysis of multiple cytosine-guanine dinucleotide (CpG) sites was performed by the Beijing Genomics Institute (Guanzhou, China) using a Sequenom EpiTYPE system based on MALDI-TOF mass spectrometry according to Suchiman et al [20]. The CpG-rich sequences for MYF5 and MYOD promoters were selected using EMBOSS Cpgplot (https://www.ebi.ac.uk/Tools/seqstats/emboss_cpgplot/), and one $\mathrm{CpG}$ island was found in the promoter regions of MYF5 and MYOD. The CpG island of the MYF5 promoter ranges from -10 to $+347 \mathrm{bp}$ relative to the transcriptional start site (TSS) (10070240-10070597 on chromosome 5). The forward primer sequence of MYF5 was TTTATTTTGGG TAGTTTTTGGTTAGG tagged with the T7 promoter sequence aggaagagag, while the reverse sequence was CCCA AAAATATATAAAAAACCCCAA tagged with the sequence cagtaatacgactcactatagggagaaggct. The product size was 558

Table 1. Primer sequences used for quantitative real-time polymerase chain reaction

\begin{tabular}{|c|c|c|c|c|}
\hline Gene & Forward ( $5^{\prime}$ to $\left.3^{\prime}\right)$ & Reverse (5' to $3^{\prime}$ ) & Product length (bp) & Locus \\
\hline $\mathrm{MyHCl}$ & ACCAACCTGTCCAAGTTCCG & CGCGGCTACTCCTCATTCAA & 143 & XM_018054604.1 \\
\hline MyHCIIA & AAGGGCTGACATTGCTGAGT & TGCCTCTCTTCAGTCATTCCA & 122 & XM_018064659.1 \\
\hline MyHCIIX & GGTCTACGCAAACACGAGAG & GCGGAATTTGGAGAGGTTGAC & 177 & XM_018064657.1 \\
\hline MYF5 & AGACGCCTGAAGAAGGTCAA & CTCCACCTGTTCCCTTAGCA & 150 & NM_001287037.1 \\
\hline MYF6 & CAAGTCAGAGGCCAAGGAAG & TTCTAAGGGCTGCAGGGTAA & 103 & NM_001285602.1 \\
\hline MYOD & TGCAAACGCAAGACGACTAA & CTGGTTTGGGTTGCTAGACG & 126 & XM_018058990.1 \\
\hline MYOG & ACAATCTGCACTCССТСАСC & CATCCTGGCAGACAATCTCA & 106 & NM_001285733.1 \\
\hline EYA1 & CСACTCATGTCCAGCTCAGA & GACTGCGAGGCTGTTAAACC & 137 & XM_013967225.2 \\
\hline SIX1 & CAGTCACCTCGCACTTTGAA & TCCTTCATTTCCCACAGAGG & 160 & XM_018058424.1 \\
\hline MYOZ1 & GGACAGCAATGCCTTATGGT & AACTAAGGGTTCGCTCAGCA & 101 & XM_005699215.3 \\
\hline MYOZ2 & TGCCATGCAGAATGAGAAAC & TAGGGACAGCTGTGGTGTTG & 182 & XM_013964451.2 \\
\hline ACTG1 & ATGGCTACTGCTGCGTCGT & TTGAAGGTGGTCTCGTGGAT & 161 & XM_018063603.1 \\
\hline
\end{tabular}

$\mathrm{MyHCl}$, myosin heavy chain 1; MyHCIIA, myosin heavy chain IIA; MyHCIIX, myosin heavy chain IIX; MYF5, myogenic factor 5; MYF6, myogenic factor 6; MYOD, myogenic differentiation 1; MYOG, myogenin; EYA1, eyes absent homolog 1; SIX 1, homeobox protein SIX1; MYOZ1, myozenin 1; MYOZ2, myozenin 2; ACTG1, actin gamma 1 
bp from -44 to +503 (10070196 to 10070753 on chromosome 5) and covered $41 \mathrm{CpG}$ sites, among which $34 \mathrm{CpGs}$ could be effectively quantified. The $\mathrm{CpG}$ island of the MYOD promoter ranges from +159 to +888 relative to the TSS site (47532862 to 47533591 on chromosome 15). The forward primer sequence of MYOD was TAGTTTTGGGAGTTTA GTGTGAAGG tagged with the T7 promoter sequence agga agagag, while the reverse sequence was CCTTACAAACCC ACAATAAACAA was tagged with cagtaatacgactcactatag ggagaaggct. The product size was $546 \mathrm{bp}$ from -15 to +530 (47532688-47533233 on chromosome 15) and covered 55 CpG sites, among which 42 CpG sites could be effectively quantified. The spectral data were preprocessed and analyzed according to the method of Suchiman et al [20]. The methylation level of the restricted group in each CpG site was expressed as the value relative to the control group.

\section{Statistical analysis}

Data were analyzed by a mixed model with treatment, gen- der, and litter size as fixed factors, and initial BW of dams as the covariate. Statistical significance was considered at $\mathrm{p}<0.05$, and the Sidak method was applied to compare mean values. All results are presented as the mean and standard errors.

\section{RESULTS}

Body weight and area of muscle fibers

The effect of maternal undernutrition on the muscle fiber area of fetuses and kids is presented in Figure 1. The BW of fetuses was unaffected $(p>0.05)$ by undernutrition, but the BW of restricted kids was decreased relative to controls ( $\mathrm{p}=$ 0.027).

The muscle fiber area of the vastus muscle of restricted fetuses was increased $(p=0.032)$, while it was unaffected $(p>$ 0.05 ) in the ST and VL muscles of restricted kids. The Gender and litter size did not affect the area of muscle fibers in the fetuses or kids $(\mathrm{p}>0.05)$.
(A)

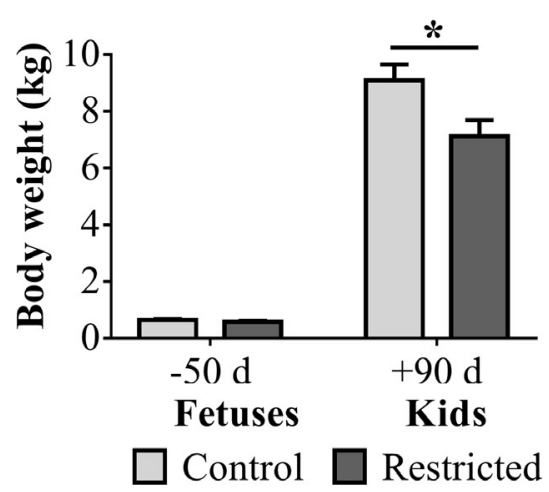

(D)

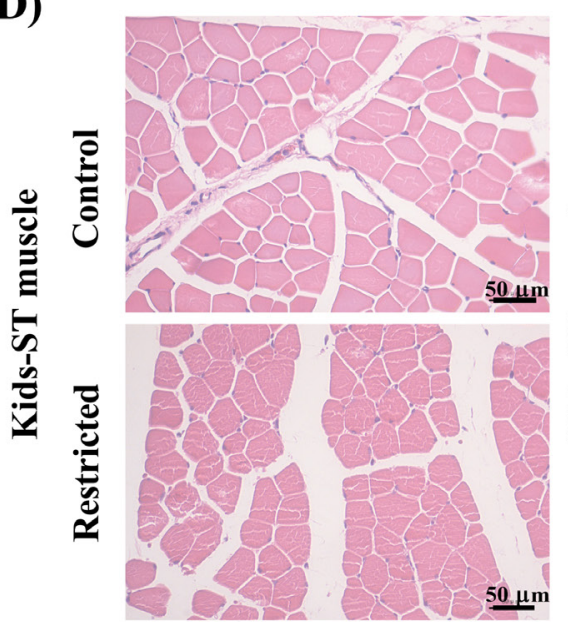

(B)

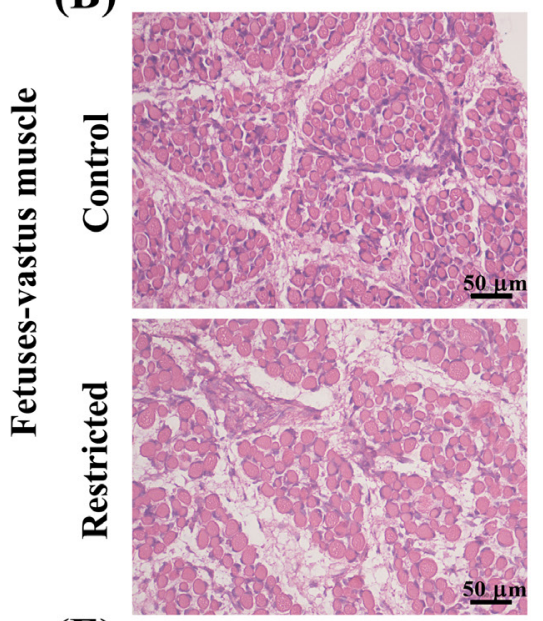

(E)

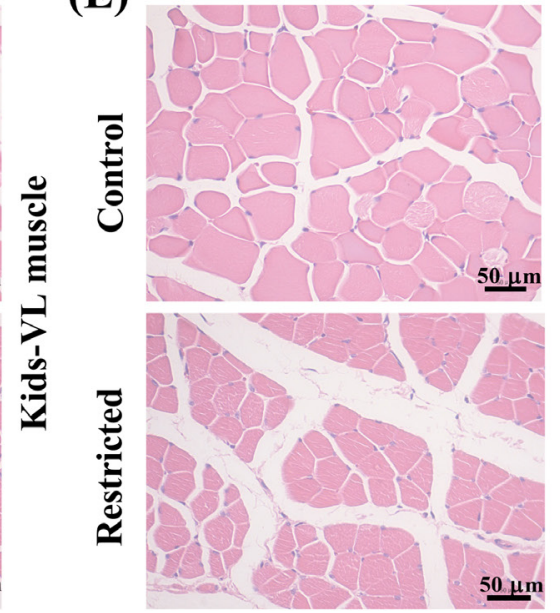

(C)

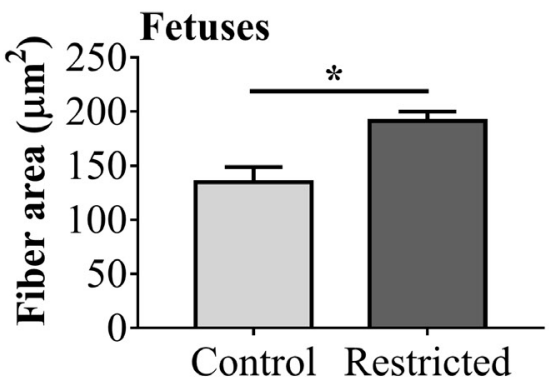

(F)

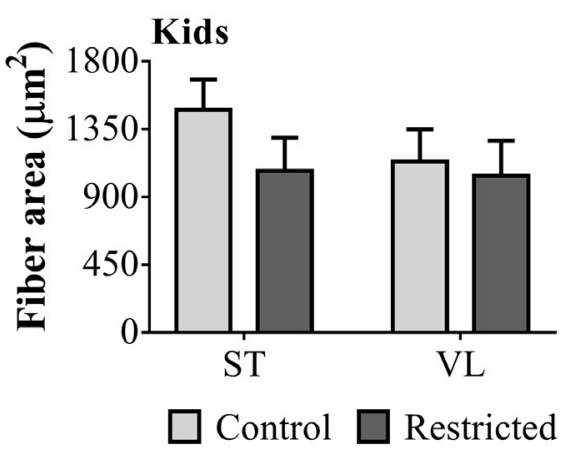

Figure 1. Body weight and muscle fiber area in offspring. (A) Body weight; (B) histological section or (C) fiber area of vastus muscle in fetuses; (D) histological section of semitendinosus (ST) muscle or (E) vastus lateralis (VL) muscle in kids; (F) fiber area of ST and VL muscles in kids. * $p<0.05$. 
Myosin heavy chain expression

The effect of maternal undernutrition on the expression of myosin heavy chain $(\mathrm{MyHC})$ in the muscle tissues of fetuses and kids is presented in Figure 2. The expression of $\mathrm{MyHCI}$ $I B$ mRNA in the fetuses and kids was not detectable, so the expression of MyHCIIB protein in these samples was not analyzed further. Compared to the control group, maternal undernutrition did not change $(\mathrm{p}>0.05)$ the expression of MyHCI, MyHCIIA, or MyHCIIX mRNA in the vastus muscles of fetuses (Figure 2A) or in the ST (Figure 2B) and VL (Figure 2C) muscles of kids. However, the protein expression levels of MyHCI and MyHCIIA were increased $(\mathrm{p}<0.05)$ both in the vastus muscles of fetuses (Figure 2D) and in the ST muscles of kids (Figure 2E), while the MyHCIIX protein expression in the fetuses and in the ST muscles of kids was decreased $(\mathrm{p}<0.01)$. Protein expression levels of MyHCI, MyHCIIA, and MyHCIIX in the VL muscles of kids (Figure
$2 F)$ were not affected ( $p>0.05)$. Gender and litter size did not affect ( $\mathrm{p}>0.05$ ) the mRNA or protein expression of $\mathrm{MyHCI}$, $M y H C I I A$, and MyHCIIX genes in the fetuses or kids.

mRNA expression and DNA methylation of myogenic factors

Since significant differences in myosin heavy chain protein expression in muscle resulting from maternal undernutrition were observed, we further determined the mRNA expression and DNA methylation of myogenic factors in the muscles of fetuses and kids (Figure 3). Maternal undernutrition did not influence ( $\mathrm{p}>0.05$ ) the expression of MYF5, MYF6, MYOD, or MYOG mRNA in the vastus muscles of fetuses (Figure 3A) or in the ST muscles of kids (Figure 3B). The expression levels of eyes absent homolog $1(E Y A 1)(\mathrm{p}=0.044)$ and myozenin 2 (MYOZ2) $(\mathrm{p}=0.038)$ mRNA in the vastus muscles of restricted fetuses were downregulated, while no difference was
(A)

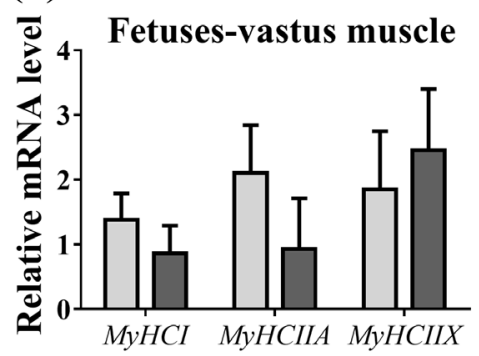

$\square$ Control $\square$ Restricted

(B)

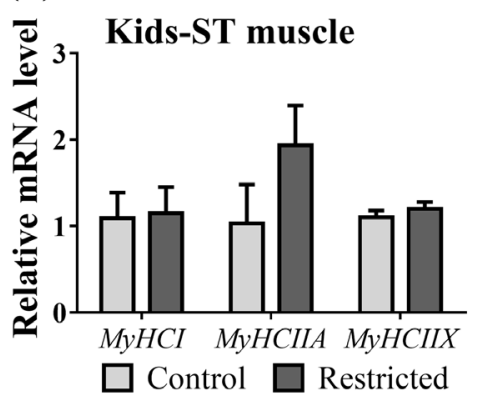

(C)

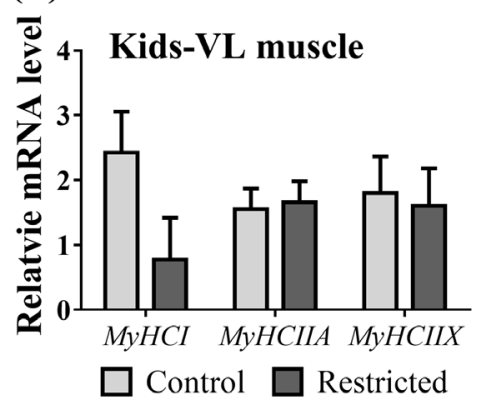

(D)

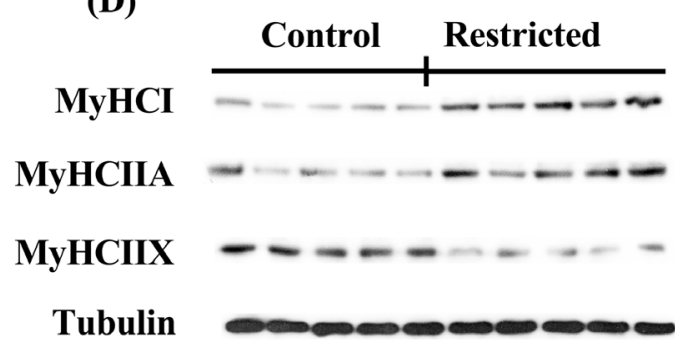

(E)

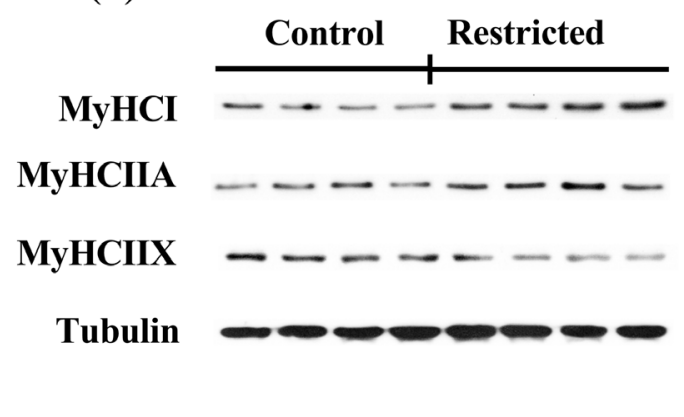

(F)

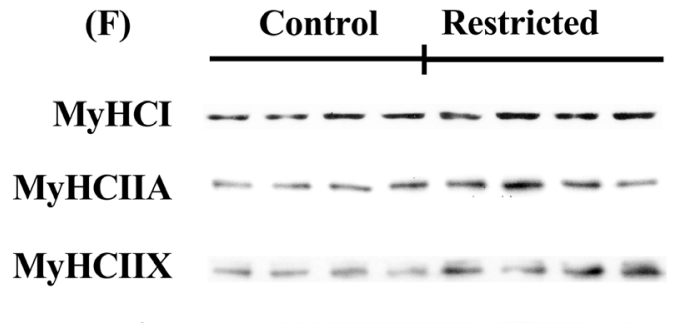

Tubulin - - - -
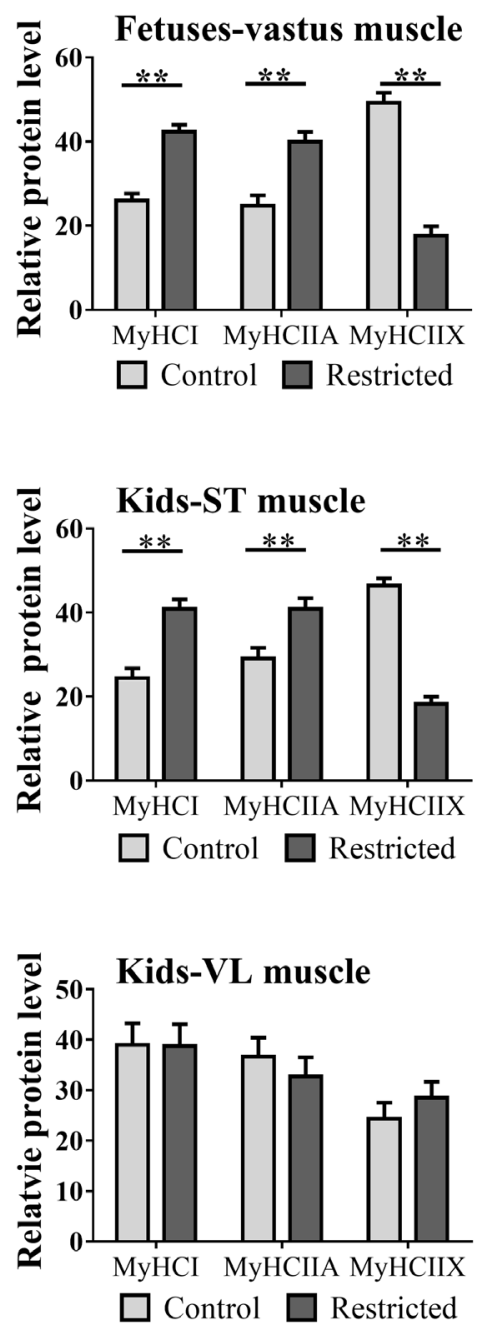

Figure 2. The mRNA and protein expression of myosin heavy chain in offspring. (A) mRNA expression of vastus muscle in fetuses; (B) mRNA expression of semitendinosus (ST) or (C) vastus lateralis (VL) muscle in kids; (D) protein expression of vastus muscle in fetuses; (E) protein expression of ST or (F) VL muscle in kids. ** $p<0.01$. 

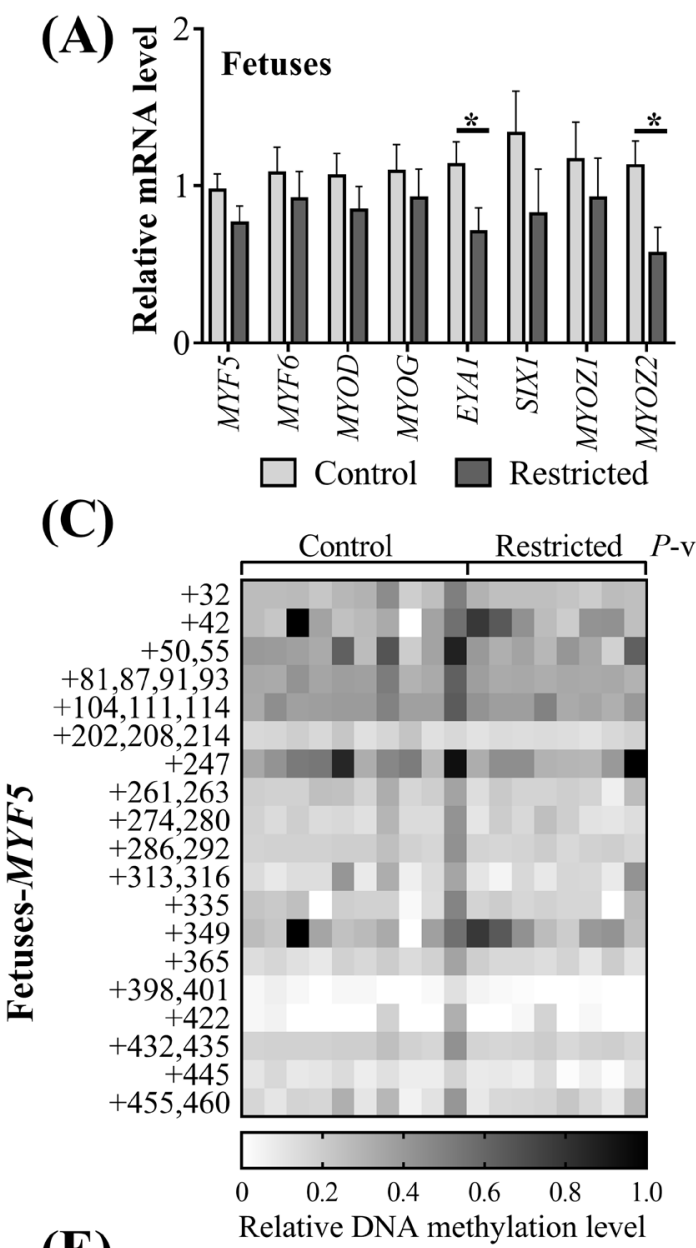

(E)

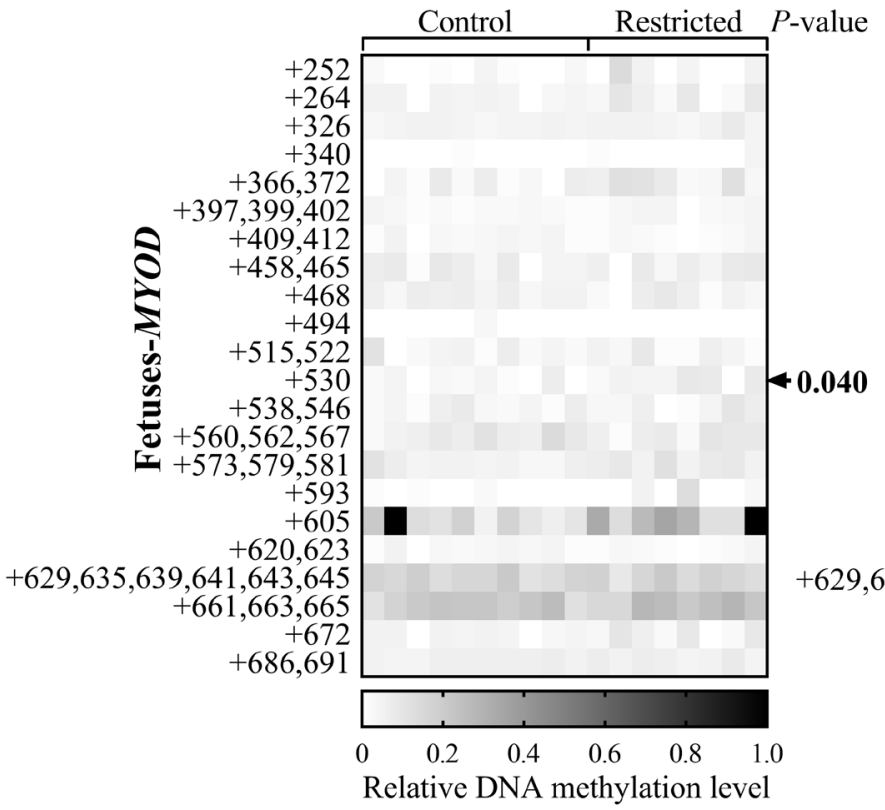

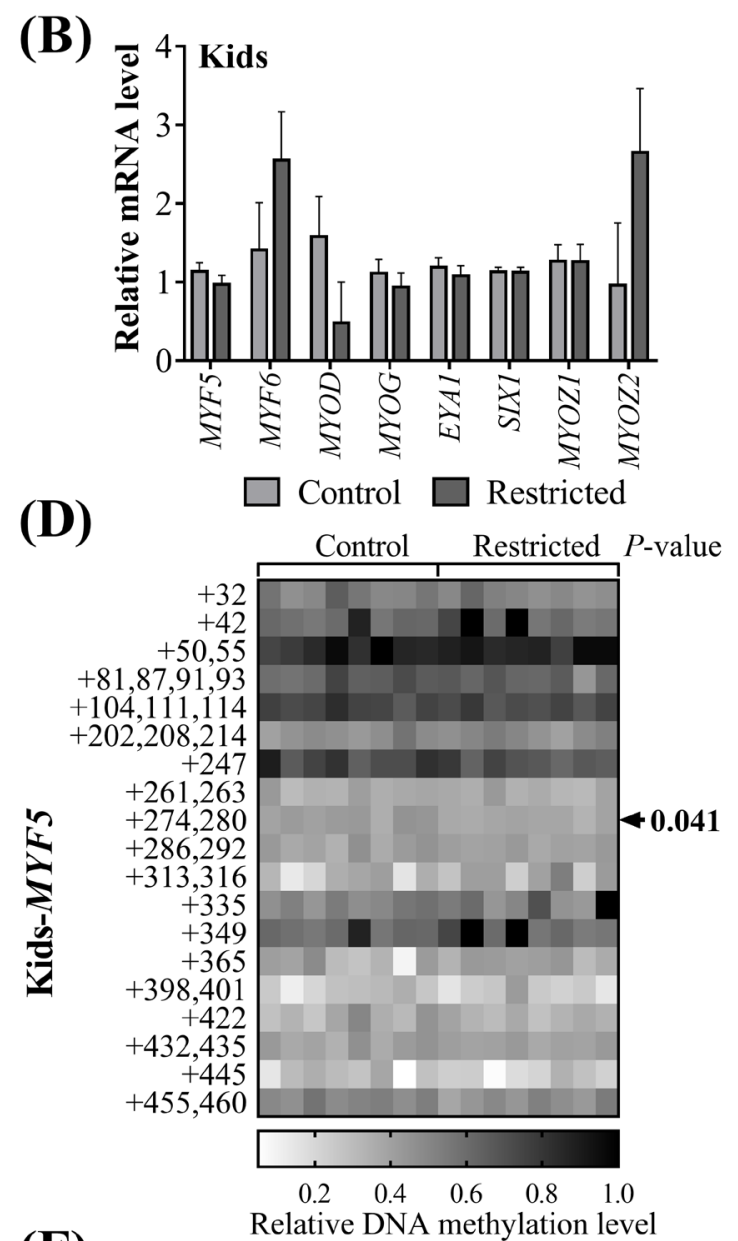

(F)

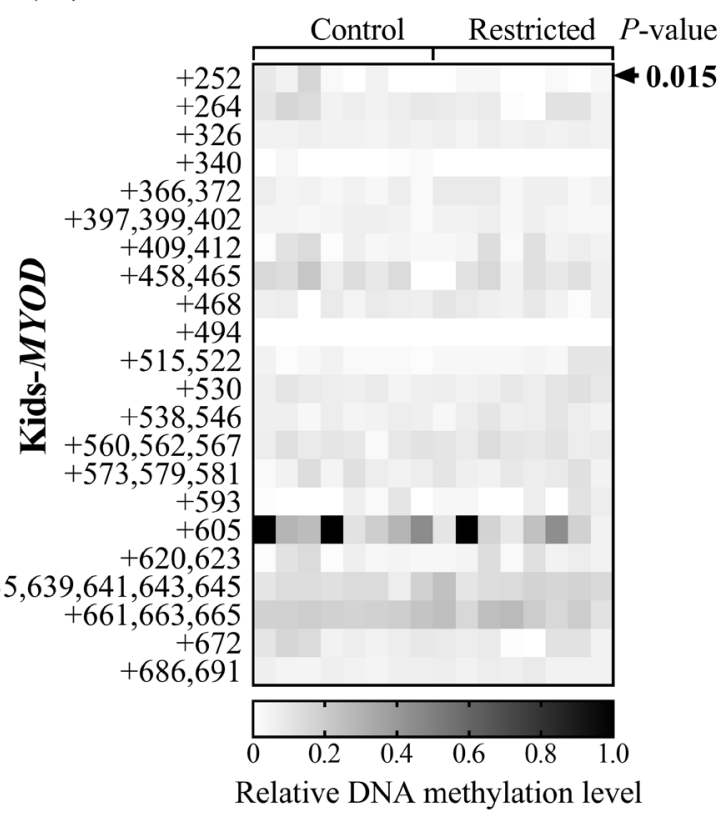

Figure 3. The mRNA expression of myogenic genes and $\mathrm{CpG}$ methylation of myogenic promoters in offspring. (A) mRNA expression of vastus muscle in fetuses; (B) mRNA expression of semitendinosus (ST) muscle in kids; methylation of CpG sites in the (C) MYF5 or (E) MYOD promoter region relative to the TSS site in vastus muscle of fetuses; methylation of CpG sites in the (D) MYF5 or (F) MYOD promoter region relative to the TSS site in the semitendinosus (ST) muscle of kids. MYF5, myogenic factor 5; MYOD, myogenic differentiation. * $p<0.05$. 
observed in the ST muscles of kids ( $\mathrm{p}>0.05)$. The methylation level of the CpG island of the MYF5 promoter was not affected ( $>0.05$ ) in the fetuses (Figure 3C). Maternal undernutrition increased $(\mathrm{p}=0.040)$ the methylation level of the $+530 \mathrm{CpG}$ site of the MYOD promoter in the fetuses (Figure $3 \mathrm{D}$ ), while the methylation levels of the $+274,280 \mathrm{CpG}$ site of the MYF5 promoter (Figure 3E) and the $+252 \mathrm{CpG}$ site of the $M Y O D$ promoter (Figure $3 \mathrm{~F}$ ) in the restricted kids were decreased $(\mathrm{p}<0.05)$. Gender and litter size did not affect $(\mathrm{p}>$ 0.05 ) the mRNA expression of these myogenic genes or the CpG methylation of MYF5 and MYOD promoters in the fe- tuses. Gender affected $(\mathrm{p}<0.05)$ the MYF5 and SIX homeobox 1 (SIX1) mRNA expression in kids; both was greater in females than in males. The methylation of the $+32 \mathrm{CpG}$ site in the MYF5 promoter of the female kids was higher $(\mathrm{p}=0.041)$ than in the males.

mTOR signaling pathway

Maternal undernutrition reduced $(\mathrm{p}<0.01)$ the protein expression of mTOR, $\mathrm{p}-\mathrm{mTOR}$, and the ratio of $\mathrm{p}-\mathrm{mTOR} / \mathrm{mTOR}$ in fetal vastus muscle (Figure 4A). Maternal undernutrition also reduced $(\mathrm{p}=0.037)$ the $\mathrm{mTOR}$ protein expression in

(A)
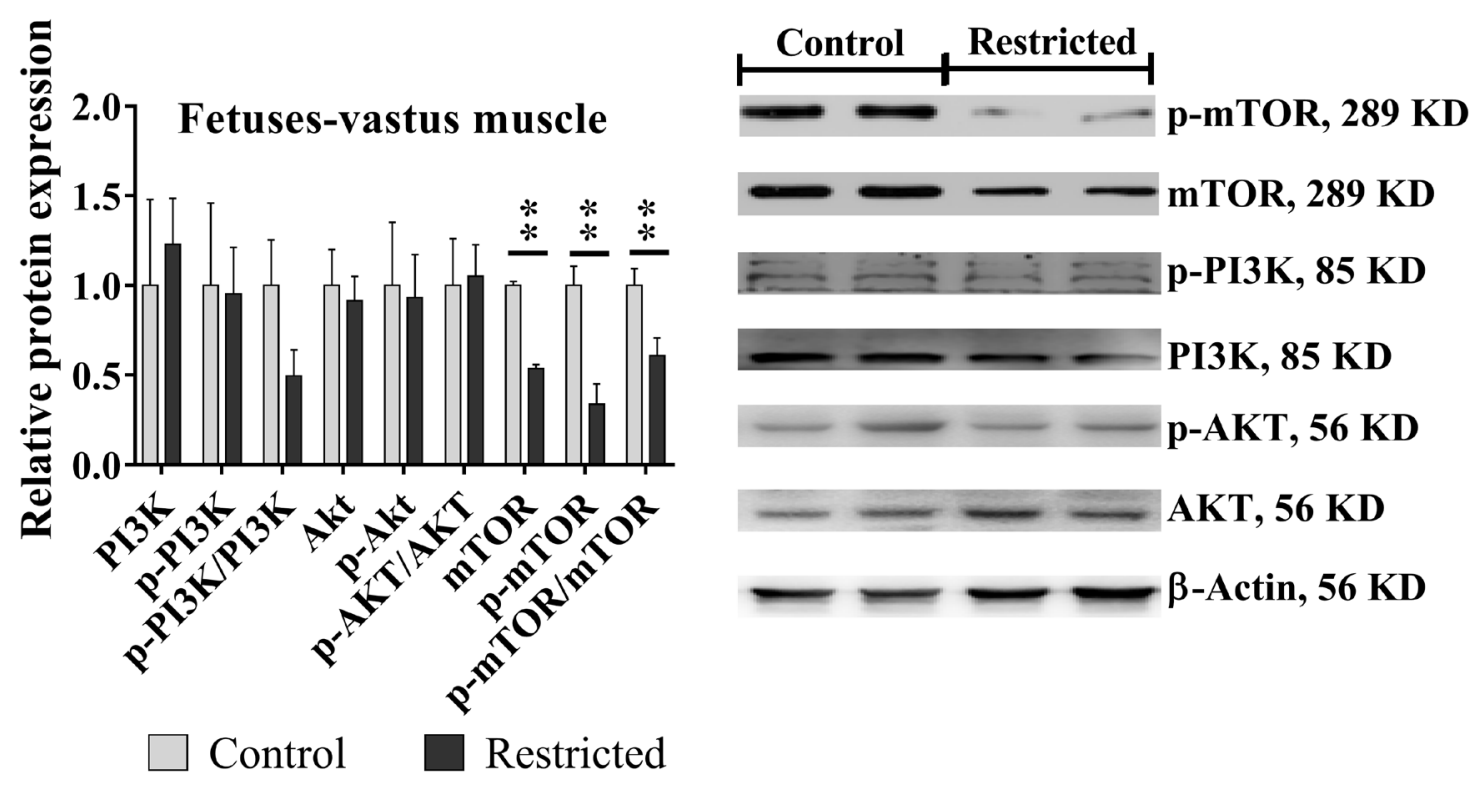

(B)
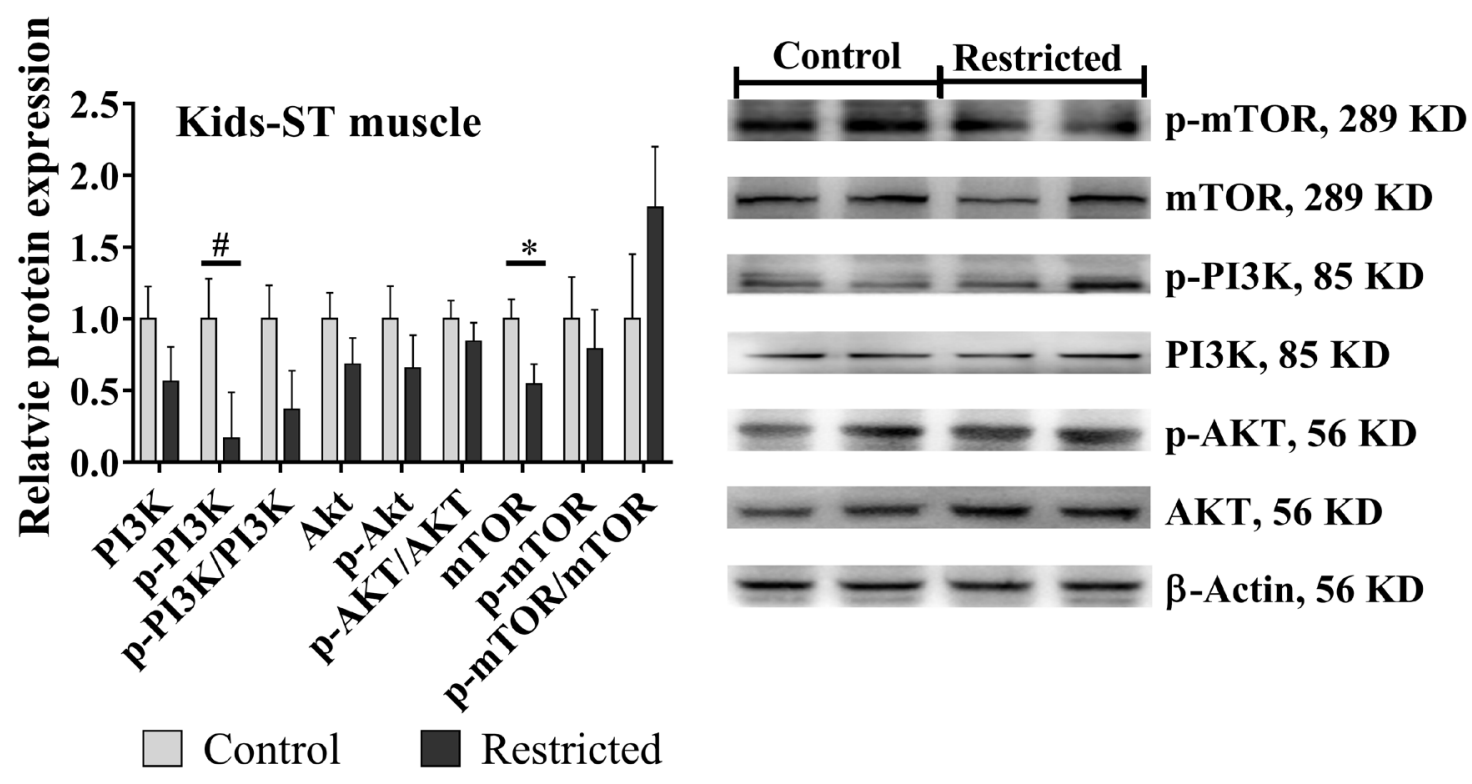

Figure 4. The protein expression of mammalian target of rapamycin (mTOR) signaling in the skeletal muscles of offspring. (A) Vastus muscles in fetuses; (B) semitendinosus (ST) muscles in kids. ${ }^{*} 0.05<p<0.10 ;{ }^{*} p<0.05 ; * \star p<0.01$ 
kids and tended to reduce $(\mathrm{p}=0.088)$ the PI3K phosphorylation level (Figure 4B), while the effects on the PI3K, AKT, and $\mathrm{p}$-AKT proteins and the ratio of $\mathrm{p}$-AKT/AKT in kids were not significant $(\mathrm{p}>0.05)$. Gender and litter size did not affect the above protein expression in the fetuses or kids ( $\mathrm{p}>$ 0.05).

\section{DISCUSSION}

During the past two decades, there has been increased research interest in the effects of maternal undernutrition on muscle development in offspring. In this study, maternal undernutrition altered the muscle fibers and MyHC types of the vastus muscle in fetuses and kids, and downregulated the mRNA expression of myogenic factors in the fetuses. DNA methylation levels of several sites in the MYF5 and $M Y O D$ promoter regions in the fetuses and kids were also affected by maternal undernutrition. Several studies have confirmed that maternal undernutrition in the second trimester is closely associated with the reduced weight of offspring in human epidemiological surveys [21] and in animal model trials $[8,22]$. In the present study, no changes in the BW of fetuses and the diminished BW in restricted kids were consistent with a compensation effect by the mother in utero and a thrifty phenotype after birth. However, the increase in muscle fiber area in restricted fetuses in this study was not consistent with the findings (no significant change) in the fetuses of cattle [12] and pigs [11] during midgestation or in sheep (a decreased size) [7]. Whether this phenomenon is due to species differences or other physiological factors needs more in-depth research. Notably, the perimysium area of the ST and VL muscles seemed larger in the restricted kids. This fraction contains a mixture of mesenchymal stem or stromal cells, fibroblasts, immune cells and endothelial cells [23]. Regrettably, we did not carry out in-situ sampling. The process of cutting muscle after slaughter may cause displacement between muscle bundles, so it is difficult to judge whether the thicker perimysium is caused by the experimental treatment or muscle sampling, hence no statistical analysis of this data has been carried out.

Skeletal muscle is generally divided into four types according to MyHC isoforms: slow-twitch type I (MyHCI) and fast-twitch types IIA (MyHCIIA), IIB (MyHCIIB), and IIX (MyHCIIX). Previously, Zhu et al [9] reported that 50\% maternal undernutrition increased the MyHCIIB ratio and decreased the percentage of MyHCIIA of the longissimus dorsi (LD) muscle in restricted lambs at eight months of age, while the MyHC type of LD and ST muscles in 50\% restricted lambs at 150 days of age was unaffected [8]. In contrast, Fahey et al [24] reported fewer fast fibers and more slow fibers in the LD and VL muscles of $50 \%$ maternally restricted 14-day-old lambs. In this study, the types of MyHCI and MyHCIIA were increased, but MyHCIIX was decreased both in the vastus muscle of $40 \%$ restricted fetuses and in the ST muscles of restricted kids, while the VL muscles in offspring were not affected. These results suggest that the effects of similar maternal undernutrition during midgestation on muscle fiber development may vary, and the cause needs further research. Moreover, comparing LD and ST fibers from the same muscle position, a similar degree of feeding restriction (such as 50\%) exerted different effects on muscle fiber development $[8,9,24]$. It has been speculated that this discrepancy is related not only to the MyHC types in different muscle positions but also to the timing of phenotypic plasticity induced by different types of nutrient restriction [25].

In this study, we noted an inconsistency in the expression between the mRNA and protein levels of MyHC isoforms in the fetal vastus and kid ST muscles. Similar differences in mRNA and protein abundances of $M y H C$ genes have been reported in other species [26]. Generally, the protein accretion rate of hindlimb muscle is approximately $0.17 \%$ per day in ovine fetuses [27] and $0.8 \%$ in young lambs [28]. However, transcriptional and posttranscriptional regulation of $\mathrm{MyHC}$ isoform mRNA in muscle tissue is active and sensitive to environmental cues (e.g., hormones or metabolites) [5,10]. MyHC expression at the protein level represents the existing fiber type within the muscle. Therefore, the effect of protein expression is highlighted.

We observed changes in the MyHC types of vastus muscle in goat offspring. The results hinted that metabolic programming could have occurred in the present study. Hence, we determined the effect on the DNA methylation of promoter sites in the MYF5 and MYOD genes. DNA methylation is one of the universal mechanisms regulating this type of phenotypic change [17]. MYF5 and MYOD gene methylation is important in regulating muscle differentiation. Increased methylation of three important $\mathrm{CpG}$ sites in the MYOD gene and decreased MYOD mRNA expression have been observed during the differentiation of human primary muscle fibers from myoblasts to myotubes, while the methylation and mRNA levels of the MYF5 gene were decreased [29]. However, there are few reports on the methylation modification of these two myogenic regulatory factors under intrauterine nutrition restriction. Our results revealed that maternal nutritional restriction during midgestation affected the methylation of individual sites in the MYOD and MYF5 promoter regions of the fetuses and kids. These affected sites may not be closely correlated with the regulation of $M Y O D$ and MYF5 mRNA expression, because the mRNA expression levels of MYOD and MYF5 were unaffected. Previous studies have shown that maternal low-amino acid diets in the middle and late stages of pregnancy reduced the methylation level of the CpG island in insulin like growth 
factor 2 receptor (IGF2R) gene intron 2 in the longissimus muscles of sheep fetuses at 130 days [30]. A similar study found that the average $\mathrm{CpG}$ island methylation in the PGC1A promoter sequence in the skeletal muscle of intrauterine growth retardation rats with protein restriction during pregnancy increased, while glucose transporter type 4 and PGC1A mRNA expression and glucose tolerance decreased [16]. In this study, we have not yet identified the key methylation modification sites that control the phenotypic changes in offspring; thus, further research is needed.

The mRNA expression levels of the myogenic factors $M Y O G$ and MRF4 in fetuses and kids were unaffected, but the mRNA levels of EYA1 and MYOZ2 were decreased in the restricted fetuses. The SIX1 and EYA1 genes are specifically involved in the differentiation of limb buds [31]. The SIX1 and EYA1 proteins accumulate preferentially in the nuclei of fast-twitch muscles and induce a fiber-type transition with the replacement of myosin heavy chain I and IIA isoforms by the faster IIB and/or IIX isoforms [32]. Furthermore, MYOZ2 is expressed in slow-twitch skeletal muscle, and the downregulation of MYOZ2 is associated with increased slow-twitch muscle fibers [33]. The downregulation of EYA1 and MYOZ2 mRNA in restricted fetuses is consistent with the increases in MyHCI and MyHCIIA types and the decrease in MyHCIIx in the present study.

The development of skeletal muscle is a highly complicated process involving nutritional factors and numerous signaling pathways that regulate muscle-specific transcription factors, and mTOR signaling is a key regulator of skeletal muscle development at distinct stages of myogenesis [34]. In this study, the mTOR protein was downregulated in the femoris muscles of fetuses and kids. We considered that compared to the normal energy requirement, $40 \%$ maternal undernutrition aggravated the overall lack of proteins and amino acids, resulting in the downregulation of mTOR signals in the offspring, thereby affecting the protein synthesis of muscle tissues. Furthermore, the mTOR protein controls muscle-specific miR-1 transcription in mice, which regulates the stability of MYOD and MYF5 expression [35]. In addition, other miRNAs such as miR-133, miR-206, and miR125b are also linked to the mTOR pathway to regulate muscle fiber differentiation [35], and the muscle-specific expression of miRNAs can be regulated by nutrients through epigenetic mechanisms [34]. This provides new ideas for studying the mechanism of muscle fiber development programming under maternal nutrition restriction.

\section{CONCLUSION}

Maternal undernutrition during midgestation increased the expression of MyHCI and MyHCIIA proteins in the vastus muscles of fetuses and the ST muscles of kids and reduced
MyHCIIX protein expression, hinting at the developmental programming of skeletal muscle fibers. Transcripts of EYA1 and MYOZ2 genes in restricted fetuses were downregulated, and $\mathrm{mTOR}$ protein signals declined in restricted fetuses and kids. Maternal feeding restriction may prompt the switch from MyHCIIX to MyHCI and IIA in the femoris muscles of fetuses and kids associated with repression of the mTOR pathway. This programming was associated with the reduction of EYA1 and MYOZ2 transcripts, but the association between mRNA expression and DNA methylation in the promoter region of the myogenic factors MYF5 and MYOD needs to be further investigated.

\section{CONFLICT OF INTEREST}

We certify that there is no conflict of interest with any financial organization regarding the material discussed in the manuscript.

\section{FUNDING}

This work was supported by the National Natural Science Foundation (the People's Republic of China; 31760678 , 31730092, and 31402105); and Hunan Innovative Province Construction Project (the People's Republic of China; 2019 RS3021).

\section{SUPPLEMENTARY MATERIAL}

Supplementary file is available from: https://doi.org/10.5713/ ab.21.0285

Supplementary Table S1. Ingredients and composition of experimental diets for dams and kids

\section{REFERENCES}

1. Marini M, Manetti M, Rosa I, Ibba-Manneschi L, Sgambati E. Telocytes in human fetal skeletal muscle interstitium during early myogenesis. Acta Histochem 2018;120:397-404. https:// doi.org/10.1016/j.acthis.2018.04.003

2. Fahey AJ, Brameld JM, Parr T, Buttery PJ. Otogeny of factors associated with proliferation and differentiation of muscle in the ovine fetus. J Anim Sci 2005;83:2330-8. https://doi. org/10.2527/2005.83102330x

3. Sharples AP, Stewart CE, Seaborne RA. Does skeletal muscle have an 'epi'-memory? The role of epigenetics in nutritional programming, metabolic disease, aging and exercise. Aging Cell 2016;15:603-16. https://doi.org/10.1111/acel.12486

4. Du M, Wang B, Fu X, Yang Q, Zhu MJ. Fetal programming in meat production. Meat Sci 2015;109:40-7. https://doi.org/ 10.1016/j.meatsci.2015.04.010 
5. Rehfeldt C, Te Pas MFW, Wimmers K, et al. Advances in research on the prenatal development of skeletal muscle in animals in relation to the quality of muscle-based food. I. Regulation of myogenesis and environmental impact. Animal 2011;5:703-17. https://doi.org/10.1017/S1751731110002089

6. Wigmore PM, Stickland NC. Muscle development in large and small pig fetuses. J Anat 1983;137:235-45.

7. Zhu MJ, Ford SP, Nathanielsz PW, Du M. Effect of maternal nutrient restriction in sheep on the development of fetal skeletal muscle. Biol Reprod 2004;71:1968-73. https://doi. org/10.1095/biolreprod.104.034561

8. Sen U, Sirin E, Ensoy U, et al. The effect of maternal nutrition level during mid-gestation on postnatal muscle fibre composition and meat quality in lambs. Anim Prod Sci 2015; 56:834-43. https://doi.org/10.1071/An14663

9. Zhu MJ, Ford SP, Means WJ, Hess BW, Nathanielsz PW, Du M. Maternal nutrient restriction affects properties of skeletal muscle in offspring. J Physiol 2006;575:241-50. https://doi. org/10.1113/jphysiol.2006.112110

10.Zhang D, Wang X, Li Y, et al. Thyroid hormone regulates muscle fiber type conversion via miR-133a1. J Cell Biol 2014;207:753-66. https://doi.org/10.1083/jcb.201406068

11.Zou T, He D, Yu B, et al. Moderate maternal energy restriction during gestation in pigs attenuates fetal skeletal muscle development through changing myogenic gene expression and myofiber characteristics. Reprod Sci 2017;24:156-67. https://doi.org/10.1177/1933719116651151

12. Jennings TD, Gonda MG, Underwood KR, Wertz-Lutz AE, Blair AD. The influence of maternal nutrition on expression of genes responsible for adipogenesis and myogenesis in the bovine fetus. Animal 2016;10:1697-705. https://doi.org/10. 1017/S1751731116000665

13.Luo H, Lv W, Tong Q, Jin J, Xu Z, Zuo B. Functional noncoding RNA During embryonic myogenesis and postnatal muscle development and disease. Front Cell Dev Biol 2021; 9:628339. https://doi.org/10.3389/fcell.2021.628339

14.Braun T, Gautel M. Transcriptional mechanisms regulating skeletal muscle differentiation, growth and homeostasis. Nat Rev Mol Cell Biol 2011;12:349-61. https://doi.org/10.1038/ nrm3118

15. Ehrlich KC, Paterson HL, Lacey M, Ehrlich M. DNA hypomethylation in intragenic and intergenic enhancer chromatin of muscle-specific genes usually correlates with their expression. Yale J Biol Med 2016;89:441-55.

16.Zeng Y, Gu P, Liu K, Huang P. Maternal protein restriction in rats leads to reduced PGC-1 $\alpha$ expression via altered DNA methylation in skeletal muscle. Mol Med Report 2013;7:30612. https://doi.org/10.3892/mmr.2012.1134

17. Miyata K, Miyata T, Nakabayashi K, et al. DNA methylation analysis of human myoblasts during in vitro myogenic differentiation: de novo methylation of promoters of musclerelated genes and its involvement in transcriptional down- regulation. Hum Mol Genet 2015;24:410-23. https://doi.org/ 10.1093/hmg/ddu457

18.Zhou X, Yang H, Yan Q, et al. Evidence for liver energy metabolism programming in offspring subjected to intrauterine undernutrition during midgestation. Nutr Metab (Lond) 2019;16:20. https://doi.org/10.1186/s12986-019-0346-7

19.Chen W, Yan Q, Yang H, Zhou X, Tan Z. Effects of restrictions on maternal feed intake on the immune indexes of umbilical cord blood and liver Toll-like receptor signaling pathways in fetal goats during pregnancy. J Anim Sci Biotechnol 2019; 10:29. https://doi.org/10.1186/s40104-019-0336-7

20.Suchiman HED, Slieker RC, Kremer D, Slagboom PE, Heijmans BT, Tobi EW. Design, measurement and processing of regionspecific DNA methylation assays: the mass spectrometrybased method EpiTYPER. Front Genet 2015;6:287. https:// doi.org/10.3389/fgene.2015.00287

21.Retnakaran R, Wen SW, Tan H, et al. Association of timing of weight gain in pregnancy with infant birth weight. JAMA Pediatr 2018;172:136-42. https://doi.org/10.1001/jamape diatrics.2017.4016

22.Piaggio L, Quintans G, San Julian R, et al. Growth, meat and feed efficiency traits of lambs born to ewes submitted to energy restriction during mid-gestation. Animal 2018;12: 256-64. https://doi.org/10.1017/S1751731117001550

23.Li X, Fu X, Yang G, Du M. Review: Enhancing intramuscular fat development via targeting fibro-adipogenic progenitor cells in meat animals. Animal 2020;14:312-21. https://doi. org/10.1017/S175173111900209X

24. Fahey AJ, Brameld JM, Parr T, Buttery PJ. The effect of maternal undernutrition before muscle differentiation on the muscle fiber development of the newborn lamb. J Anim Sci 2005;83: 2564-71. https://doi.org/10.2527/2005.83112564x

25. Wells JCK. Developmental plasticity as adaptation: adjusting to the external environment under the imprint of maternal capital. Philos Trans R Soc Lond B Biol Sci 2019;374:20180122. https://doi.org/10.1098/rstb.2018.0122

26. Sun X, Liu Z, Wu B, et al. Differences between fast and slow muscles in scallops revealed through proteomics and transcriptomics. BMC Genomics 2018;19:377. https://doi.org/ 10.1186/s12864-018-4770-2

27. Rozance PJ, Zastoupil L, Wesolowski SR, et al. Skeletal muscle protein accretion rates and hindlimb growth are reduced in late gestation intrauterine growth-restricted fetal sheep. J Physiol 2018;596:67-82. https://doi.org/10.1113/jp275230

28. Claeys MC, Mulvaney DR, McCarthy FD, et al. Skeletal muscle protein synthesis and growth hormone secretion in young lambs treated with clenbuterol. J Anim Sci 1989;67:2245-54. https://doi.org/10.2527/jas1989.6792245x

29. Davegårdh C, Broholm C, Perfilyev A, et al. Abnormal epigenetic changes during differentiation of human skeletal muscle stem cells from obese subjects. BMC Med 2017;15: 39. https://doi.org/10.1186/s12916-017-0792-x 
30.Lan X, Cretney EC, Kropp J, et al. Maternal diet during pregnancy induces gene expression and dna methylation changes in fetal tissues in sheep. Front Genet 2013;4:49. https://doi. org/10.3389/fgene.2013.00049

31. Fougerousse F, Durand M, Lopez S, et al. Six and Eya expression during human somitogenesis and $\mathrm{MyoD}$ gene family activation. J Muscle Res Cell Motil 2002;23:255-64. https:// doi.org/10.1023/A:1020990825644

32. Grifone R, Laclef C, Spitz F, et al. Sixl and Eyal expression can reprogram adult muscle from the slow-twitch phenotype into the fast-twitch phenotype. Mol Cell Biol 2004;24:625367. https://doi.org/10.1128/mcb.24.14.6253-6267.2004

33. Frey N, Barrientos T, Shelton JM, et al. Mice lacking calsarcin-1 are sensitized to calcineurin signaling and show accelerated cardiomyopathy in response to pathological biomechanical stress. Nat Med 2004;10:1336-43. https://doi.org/10.1038/ $\mathrm{nm} 1132$

34.Zhang Y, Yu B, He J, Chen D. From Nutrient to microRNA: a novel insight into cell signaling involved in skeletal muscle development and disease. Int J Biol Sci 2016;12:1247-61. https:// doi.org/10.7150/ijbs.16463

35. Sun Y, Ge Y, Drnevich J, Zhao Y, Band M, Chen J. Mammalian target of rapamycin regulates miRNA- 1 and follistatin in skeletal myogenesis. J Cell Biol 2010;189:1157-69. https:// doi.org/10.1083/jcb.200912093 